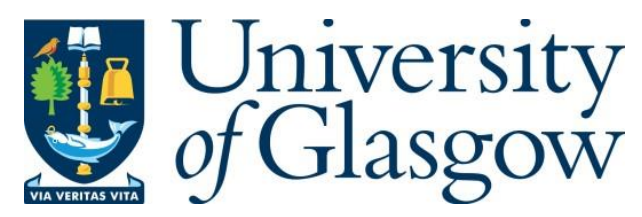

Lindner, A. K. et al. (2020) New WHO guidelines for treatment of gambiense human African trypanosomiasis including fexinidazole: substantial changes for clinical practice. Lancet Infectious Diseases, 20(2), e38-e46. (doi: 10.1016/S1473-3099(19)30612-7)

There may be differences between this version and the published version. You are advised to consult the publisher's version if you wish to cite from it.

http://eprints.gla.ac.uk/207479/

Deposited on: 19 February 2020

Enlighten - Research publications by members of the University of Glasgow http://eprints.gla.ac.uk 
Lindner AK, Lejon V, Chappuis F, Seixas J, Kazumba L, Barrett M, Mwamba E, Erphas O, AkI E, Villanueva G, Bergman H, Simarro PP, Kadima Ebeja A, Gerardo Priotto G, Franco Minguell JR. New WHO guidelines for treatment of gambiense human African trypanosomiasis including fexinidazole: fundamental changes for clinical practice. Lancet Infect Dis 2020; 20: e38-46

https://doi.org/10.1016/ S1473-3099(19)30612-7

\title{
New WHO guidelines for treatment of gambiense human African trypanosomiasis including fexinidazole: significant changes for clinical practice
}

Brief title: WHO treatment guideline for g-HAT

Andreas K. Lindner ${ }^{1}$, Veerle Lejon ${ }^{2}$, François Chappuis ${ }^{3}$, Jorge Seixas ${ }^{4}$, Leon Kazumba ${ }^{5}$, Michael P. Barrett $^{6}$, Erick Mwamba ${ }^{7}$, Olema Erphas ${ }^{8}$, Elie A. Akl ${ }^{9}$, Gemma Villanueva ${ }^{10}$, Hanna Bergman ${ }^{10}$, Pere Simarro $^{11}$, Augustin Kadima Ebeja ${ }^{12}$, Gerardo Priotto ${ }^{11}$ and Jose Ramon Franco Minguell ${ }^{11}$

1. Institute of Tropical Medicine and International Health, Charité - University Medicine, Berlin, Germany

2. Intertryp, Institut de Recherche pour le Développement, CIRAD, University of Montpellier, Montpellier, France

3. Hôpitaux Universitaires de Genève, Switzerland

4. Institute of Hygiene and Tropical Medicine / Global Health and Tropical Medicine R\&D Center, NOVA University, Lisbon, Portugal

5. Neurology Department, University of Kinshasa, DRC

6. Wellcome Centre for Integrative Parasitology, University of Glasgow, UK

7. National HAT Control Program, Ministry of Health, DRC

8. National HAT Control Program, Ministry of Health, Uganda

9. AUB GRADE Center; Center for Systematic Reviews in Health Policy and Systems Research (SPARK), American University of Beirut, Lebanon

10. Cochrane Response, London, UK

11. Neglected Tropical Diseases Department, World Health Organization, Geneva, Switzerland

12. World Health Organization Office for the Democratic Republic of Congo, Kinshasa, DRC

Corresponding author: Veerle Lejon, veerle.lejon@ird.fr, +33 467593950

\begin{abstract}
Gambiense human African trypanosomiasis (g-HAT) is a parasitic infection that usually progresses to coma and death unless treated. The WHO has updated its guidelines for the treatment of g-HAT based on independent literature reviews and using the GRADE methodology. The first-line treatment options, pentamidine and nifurtimox-eflornithine combination therapy (NECT), have been expanded to include fexinidazole, an oral monotherapy recently given a positive opinion from the European Medicines Agency. Fexinidazole is recommended for individuals $\geq 6$ years and $\geq 20 \mathrm{~kg}$, in first and second stage g-HAT with cerebrospinal fluid leukocytosis $<100 / \mu 1$. NECT remains recommended for those with $\geq 100$ leukocytes $/ \mu 1$. Without clinical suspicion of severe second stage, lumbar puncture
\end{abstract}


can be avoided and fexinidazole given. Fexinidazole should only be administered under supervision of trained health staff. As these recommendations are expected to change clinical practice considerably, health professionals should consult the detailed WHO guidelines. These guidelines will be updated as evidence accrues.

\section{Introduction}

Human African trypanosomiasis (HAT), or sleeping sickness, is a neglected tropical disease that afflicts populations in rural sub-Saharan Africa, where the tsetse fly vector transmits the parasite. Two forms of the disease exist: the usually slowly progressing form, known as gambiense HAT (g-HAT), caused by Trypanosoma brucei gambiense, endemic in West and Central Africa; and the usually faster progressing form, known as rhodesiense HAT, caused by Trypanosoma brucei rhodesiense, endemic in eastern and southern Africa.

After devastating epidemics during the 20th century, sustained and coordinated control efforts over the past 20 years led to a historically low number of 1446 reported cases in 2017, the vast majority of which were g-HAT (98\%). Rhodesiense HAT is mainly a zoonosis that occasionally affects humans. The target of eliminating HAT as a public health problem by 2020 with $<2000$ HAT cases/year and $90 \%$ reduction of the areas at risk (reporting $\geq 1$ case/10 000 people per year), has therefore nearly been met. ${ }^{1,2}$ This remarkable progress has relied on case-finding and treatment, a strategy that reduces transmission by depleting the parasite reservoir in humans, and has been occasionally complemented with vector control activities.

The treatment of g-HAT is stage dependent, until now requiring all patients to undergo a systematic lumbar puncture and cerebrospinal fluid (CSF) examination, to discriminate between first (hemolymphatic) and second (meningo-encephalitic) stages. ${ }^{3}$ The recommended first line treatment of first stage g-HAT ( $\leq 5$ white blood cells (WBC) $/ \mu \mathrm{L}$ and no trypanosomes in CSF) has been pentamidine. Pentamidine is given intramuscularly once daily for 7 days, and can be administered at the primary health care level. The first line treatment of second stage g-HAT ( $>5 \mathrm{WBC} / \mu \mathrm{L}$ and/or trypanosomes in CSF) has been nifurtimox (orally in three daily doses for 10 days) and eflornithine (intravenously in two daily infusions for 7 days) combination therapy (NECT). ${ }^{3}$ NECT has been a major improvement in therapy, when compared with its predecessors, melarsoprol or eflornithine monotherapy. ${ }^{4}$ However, NECT requires patient hospitalization, intensive nursing and complex drug transport logistics. ${ }^{5}$

Fexinidazole is an effective oral monotherapy against g-HAT. ${ }^{6}$ In November 2018, the European Medicines Agency (EMA) issued a positive opinion for fexinidazole treatment of g-HAT. That opinion was framed by article 58, a mechanism designed for drugs intended for use outside the EU.7, In December 2018, marketing authorization was given in the Democratic Republic of Congo which harbours most cases of g-HAT.

Fexinidazole is administrated orally once daily for ten days (four days loading dose, six days maintenance dose). There is evidence supporting its effectiveness in both disease stages. ${ }^{6}$ These features open the way for clinically significant modifications in the management of g-HAT, such as circumventing systematic lumbar puncture and removing the need for injectable treatment in specific groups of patients. However, this new drug also has limitations. First, patients with severe central nervous system involvement are at higher risk of failing treatment. ${ }^{7}$ Second, the tablets should be taken with a meal because bioavailability is seriously compromised in the unfed state. ${ }^{9}$ 
The EMA stated that fexinidazole should be used in line with official recommendations. ${ }^{7}$ In December 2018, the WHO Guideline Development Group on the treatment of HAT met in Geneva to provide updated evidence-based recommendations on therapeutic choices for policy makers and medical staff. The detailed treatment guidelines on g-HAT, which resulted from this meeting, are accessible on the WHO website. ${ }^{10}$ The objective of this paper is to document the decision process, to provide complementary information, to summarize the updated WHO recommendations, and to discuss their implications for clinical practice.

\section{Methods}

The WHO developed these guidelines following the methodology outlined in the WHO handbook for guideline development. ${ }^{11}$ The WHO secretariat formed a guidelines development group that included individuals with recognized expertise in the field of treatment of HAT, public health, and national control programs. The group was co-chaired by a content expert and a guideline methodologist.

In an initial prioritization process, key questions were formulated pertaining to g-HAT treatment and outcomes judged important to patients within the context of the disease and its setting. The questions, structured in PICO format (population, intervention, comparison, outcomes), were: ${ }^{12}$ 1) Should fexinidazole or pentamidine be used for first stage g-HAT; 2) Should fexinidazole or NECT be used for second stage g-HAT; 3) Should clinical stratification or lumbar puncture stratification or no stratification be used for the treatment of g-HAT; 4) Should inpatient administration or outpatient administration under supervision be used for the treatment with fexinidazole.

A systematic review was externally commissioned to synthesize the evidence relevant to the PICO questions. ${ }^{13}$ The full version of the guidelines provides the details of the review, including the search strategy, study selection, data extraction, and data analysis. ${ }^{14}$ The Grading of Recommendations Assessment, Development and Evaluation (GRADE) methodology was followed to rate the certainty of the evidence for each outcome as high, moderate, low, or very low. ${ }^{15,16}$ The evidence was then summarized by outcome using the 'Summary of finding' tables. ${ }^{15}$ The tables provide outcomespecific information concerning the certainty of evidence and the relevant statistical information. ${ }^{15,17}$

Following the GRADE methodology, the guideline development group shaped the recommendations and graded their strength as either strong or conditional. ${ }^{18}$ The grading considered the following factors: the desirable and undesirable effects of the intervention relative to its comparator; the overall certainty of the evidence; the values attached to the main outcomes; the balance between desirable and undesirable effects; the resource requirements; the impact on health equity; the acceptability of the intervention to key stakeholders; and the feasibility.

\section{Results}

Table 1 provides a summary of recommendations addressing the four PICO questions, their strength, the certainty of the supporting evidence, and key considerations. Detailed judgements on various factors considered when grading the recommendations are provided in the Evidence to Decision tables accessible on the WHO website. ${ }^{10}$

\section{PICO 1: Fexinidazole or pentamidine for the treatment of first stage g-HAT}

The panel suggests using fexinidazole over pentamidine in patients with first stage g-HAT (conditional recommendation, very low certainty of evidence).

So far, no clinical trial compared fexinidazole with pentamidine. Data about fexinidazole treatment of first stage patients originate from two prospective, open-label, single-arm studies in adults $(n=189)$ 
(DNDiFEX005) and children ( $\geq 6$ years old and $\geq 20 \mathrm{~kg}, \mathrm{n}=69$ ) (DNDiFEX006). Their respective findings at 18 months were as follows: ${ }^{14}$ failure rates of $2.1 \%$ and $1.4 \%$, mortality rates of $1.6 \%$ and $1.4 \%$, adverse event rates of $93.1 \%$ and $88.4 \%$, and serious adverse event rates of $9.0 \%$ and $7.2 \%$.

For pentamidine treatment of first stage g-HAT, evidence originated from the comparator arm of two randomized clinical trials ${ }^{19,20}$ and nine observational studies ${ }^{21-29}$ that in total included 6722 treated children and adults. The comparability of these studies is limited due to the heterogeneity of study populations, outcome criteria and observation periods. The range of treatment failure rates was $3.9 \%-$ 4.6\%. Adverse events occurred in $17.6 \%-98.5 \%$ of treated patients and serious adverse events in $2 \cdot 4 \%-17 \cdot 5 \% .^{14}$

The balance of desirable and undesirable effects appears to favour fexinidazole. Adverse event rates seem to be similar, but the events are of different types. Fexinidazole causes gastro-intestinal events, mainly vomiting and nausea, as well as headache, insomnia, tremor, and dizziness. The main adverse events for pentamidine are hypotension, nausea, vomiting and pain at the injection site. Information was insufficient to compare the direct costs. However, the indirect costs in terms of human resources are probably lower for oral fexinidazole treatment, than for intramuscular injection with pentamidine. Children aged $<6$ years and/or $<20 \mathrm{~kg}$ should receive pentamidine, as the safety and efficacy of fexinidazole in this age group has not been established in clinical trials.

\section{PICO 2: Fexinidazole or NECT for the treatment of second stage g-HAT}

The panel suggests using fexinidazole over NECT in patients with second stage g-HAT and CSF WBC $<100 / \mu l$ (conditional recommendation, low certainty of evidence).

The panel suggests using NECT over fexinidazole in patients with second stage g-HAT and CSF WBC $\geq 100 / \mu l$ (conditional recommendation, low certainty of evidence).

In one randomized, non-inferiority trial, 264 patients $\geq 15$ years with second stage g-HAT, having $>20$ WBC/ $\mu 1$ or trypanosomes in CSF, were treated with fexinidazole, 130 with NECT. ${ }^{6}$ Treatment failure rates at 24 months were $10 \cdot 3 \%$ with fexinidazole versus $2.4 \%$ with NECT (RR $4 \cdot 36,95 \%$ CI $1.35-$ 14.11). ${ }^{14}$ Adverse events at 18 months occurred in $94.0 \%$ of patients in the fexinidazole group versus 93.1\% for NECT (RR 1.01, 95\% CI 0.95-1.06), serious adverse events in $11.7 \%$ versus $10 \%$, respectively (RR $1 \cdot 17,95 \%$ CI $0 \cdot 64-2 \cdot 17) .{ }^{14}$ The most frequently reported adverse events were, similar as above: gastro-intestinal (60\%), headache, insomnia, asthenia, tremor and dizziness, which occurred in a higher percentage for fexinidazole, with the exception of vomiting. The EMA report highlighted that in this trial, in patients with second stage g-HAT and CSF $\geq 100 \mathrm{WBC} / \mu \mathrm{l}$ the failure rate at 18 months was significantly higher for fexinidazole (13.1\%) than for NECT (1.3\%). In contrast, in the group with CSF $<100 \mathrm{WBC} / \mu 1$ treatment failure rates with fexinidazole and NECT were similar, $2.0 \%$ and $4.1 \%$ respectively. ${ }^{7}$ Furthermore, data about fexinidazole treatment of second stage patients $\geq 15$ years with $\mathrm{CSF} \leq 20 \mathrm{WBC} / \mu$ l originated from a single-arm study $(\mathrm{n}=41)\left(\mathrm{DNDiFEX005)}\right.$, with $2.4 \%$ treatment failure at 18 months. ${ }^{14}$ The single arm study in children 6-15 years with second stage g-HAT $(\mathrm{n}=56)\left(\mathrm{DNDiFEX006)}\right.$ revealed $1.8 \%$ treatment failure. ${ }^{14} \mathrm{In}$ both studies, similar adverse events - gastro-intestinal and CNS related - were observed, as in the randomized controlled trial.

Based on the EMA results, the panel decided to consider the patient group with "severe" second stage g-HAT having $\geq 100 \mathrm{WBC} / \mu 1$ separately and to split the PICO 2 question based on this cut-off. The balance of desirable and undesirable effects did not favour either fexinidazole or NECT if CSF WBC $<100 / \mu 1$ and favoured NECT if CSF WBC $\geq 100 / \mu 1$. Fexinidazole outpatient treatment was judged more feasible, requiring relatively little resource and allowing financial savings, probably five- to tenfold. NECT requires hospitalization and complex logistics, to deliver the comparatively large volume of drugs and accessory materials required for use. Oral treatment allows patients to be treated closer to their home, including in remote or unstable settings, which accrues fewer expenses, thus increasing 
health equity. Oral treatment with fexinidazole in non-severe HAT is expected to be the preferred treatment option (from the feasibility standpoint) for both the patients and the health system, although some patients may perceive intravenous treatment as a better option in serious illness in general. ${ }^{30}$ Children aged $<6$ years and/or with $<20 \mathrm{~kg}$ should receive NECT, as fexinidazole is not approved for this group.

\section{PICO 3: Clinical stratification or lumbar puncture stratification or no stratification for the treatment of g-HAT}

The panel suggests doing a lumbar puncture with CSF examination over not doing a lumbar puncture (conditional recommendation, very low certainty of evidence). Without clinical suspicion of severe second stage, a lumbar puncture can be avoided and fexinidazole preferentially given.

Until now, selecting treatment for g-HAT has required a systematic lumbar puncture and CSF examination for staging. Fexinidazole is effective in both disease stages. However, as stated above, in severe second stage $(\mathrm{CSF} \geq 100 \mathrm{WBC} / \mu \mathrm{l})$ the risk of treatment failure is significantly higher with fexinidazole than with NECT., ${ }^{7}$ The panel therefore had to consider the potential benefit of avoiding systematic lumbar puncture versus the risk of treatment failure in severe second stage patients.

The panel suggested that in case of any clinical sign and symptom that raises suspicion of severe second stage, lumbar puncture and CSF examination should be performed. In the absence of a validated clinical tool for stratification, an ad-hoc group of clinicians and neurologists identified symptoms and signs that could be used for selection of patients likely to be in severe second stage. The following symptoms and signs, correlating with severe meningo-encephalitic g-HAT and assessable in peripheral health facilities, were identified: ${ }^{31,32}$ mental confusion, abnormal behaviour, logorrhoea, speech impairment, anxiety, tremor, motor weakness, ataxia, abnormal gait, abnormal movements, and seizures (table 2). The presence of any of these symptoms or signs should raise suspicion of severe second stage g-HAT. Although sleep disorder is very common in severe HAT, it is also frequent in non-severe HAT, thus this feature alone was not considered sufficient to be indicative. Without clinical suspicion of severe second stage, lumbar puncture can be avoided and fexinidazole preferentially given, on condition of having high confidence in appropriate follow-up to detect relapse early. Avoiding systematic lumbar puncture in a subgroup of patients for treatment stratification was judged to allow for moderate savings of human and material resources. As fexinidazole is not approved for children $<6$ years old or $<20 \mathrm{~kg}$ body weight, they require systematic lumbar puncture for disease staging.

\section{PICO 4: Inpatient or outpatient administration of fexinidazole under supervision}

The panel suggests either inpatient or outpatient administration of fexinidazole under supervision (conditional recommendation, very low certainty of evidence).

In the above-mentioned clinical trials, fexinidazole was administered as inpatient treatment. Taken without food, the fexinidazole bioavailability is 2.5-3 fold lower and the active metabolites do not reach therapeutic levels. ${ }^{9}$ Incomplete adherence to unsupervised oral treatments is commonly reported. The efficacy of fexinidazole as outpatient treatment risks being impaired through nonadherence and/or sub-therapeutic drug levels if taken without a meal. Therefore, an independent, nonsystematic search was conducted on adherence to oral malaria treatment (predominantly 3-day course), as a proxy of the expected adherence to the 10-day fexinidazole oral regimen. Four systematic reviews encompassing 133 studies reported high variability of adherence to malaria treatment, ranging from $1.5 \%$ to $100 \%$. Only one review (25 studies) calculated a pooled prevalence, yielding a $69.8 \%$ adherence. ${ }^{33-36}$ Experiencing vomiting, other adverse events, non-supervised first dose, lower education or income level, being male, and belief in traditional medicine, were among the factors associated with non-adherence to oral malaria treatment. 
Adults treated with fexinidazole reported a higher percentage of psychiatric adverse reactions (39\%), most mild to moderate, than those treated with NECT (18\%). ${ }^{6}$ One of the most frequent adverse reactions reported in adults treated with fexinidazole was vomiting (28-42\%), mostly mild to moderate without permanent treatment discontinuation under clinical trial conditions in hospitalised patients. ${ }^{6,14}$ Vomiting was more frequent in children (69\%). ${ }^{14}$ These adverse drug reactions were recognized as additional threats to the compliance with the 10-day fexinidazole course.

Administration of fexinidazole should, therefore, be done under the strict supervision of trained health staff, who must confirm that the patient is in a fed condition and who must directly observe each drug intake. The panel suggested administering fexinidazole in outpatient mode only if there is confidence in concomitant food intake, confidence in full adherence, absence of psychiatric disorders (history or acute), and if bodyweight is $\geq 35 \mathrm{~kg}$ (below $35 \mathrm{~kg}$ the dose is smaller and drug exposure margins are narrower). This can be done in hospitals or peripheral health facilities, and, in particular situations, at home. Outpatient versus inpatient treatment should be a shared decision between the patient, their family and the health staff involved. The preference of the patient (e.g. in regards to treatment related costs for travel, hospitalization), existing comorbidities, the risk of developing side effects interfering with compliance and the capacity of the healthcare system for supervised administration as outpatient should all be considered.

\section{Discussion}

The updated evidence-based recommendations on therapeutic choices for g-HAT can be summarized as follows. Fexinidazole replaces pentamidine as first line treatment in patients with first stage gHAT, and replaces NECT as first line treatment in patients with second stage g-HAT with CSF WBC $<100 / \mu 1$. Patients of $<6$ years old or $<20 \mathrm{~kg}$ bodyweight are excepted, as the safety and efficacy of fexinidazole in this age group is not established in the clinical trials and consequently, fexinidazole is not approved for this group. For patients with severe second stage g-HAT, defined by CSF WBC $\geq 100 / \mu 1$, NECT treatment is recommended. Without clinical suspicion of severe second stage, lumbar puncture can be avoided and fexinidazole given. Administration of fexinidazole should be done under the strict supervision of trained health staff.

The above recommendations introduce important changes into clinical practice. Detailed guidelines for policy makers and medical staff managing patients, which follow from the four recommendations formulated by the guideline development group, can be found in the WHO Guidelines for the treatment of g-HAT. ${ }^{10}$ The algorithm shown in figure 1 summarizes these recommendations. Once a patient has been diagnosed with g-HAT, a detailed clinical assessment by a health professional who has adequate training and capacity to raise suspicion of severe second stage g-HAT has a decisive role (table 2). A correlation of neurological signs and symptoms with increasing CSF WBC count and especially with $\geq 100 \mathrm{WBC} / \mu 1$ has been shown. ${ }^{31}$ A patient not presenting with any of these suggestive symptoms and signs, is assumed at low probability of severe meningo-encephalitic stage and a lumbar puncture can be avoided, with the exception of patients of $<6$ years old or $<20 \mathrm{~kg}$ bodyweight. Patients who do not need a lumbar puncture are treated with fexinidazole in case of high confidence in appropriate follow-up to detect relapse early. In the other patients, a CSF examination is required in order to establish the best treatment indication (figure 1). Based on the results of the CSF examination, the recommendations favour: 1) fexinidazole for patients ( $\geq 6$ years and $\geq 20 \mathrm{~kg}$ ) with $<100 \mathrm{WBC} / \mu \mathrm{l} \mathrm{CSF} ; 2)$ NECT for patients with $\geq 100 \mathrm{WBC} / \mu 1 \mathrm{CSF}$, for children ( $<6$ years or $<20 \mathrm{~kg}$ ) with $>5 \mathrm{WBC} / \mu 1$ and/or trypanosomes in CSF, or if the lumbar puncture is not done or if the CSF results are not interpretable; or 3 ) pentamidine for children ( $<6$ years or $<20 \mathrm{~kg}$ ) with $\leq 5 \mathrm{WBC} / \mu 1$ and no trypanosomes in CSF. Fexinidazole treatment should be given in the outpatient setting only when there is confidence in concomitant food intake, full adherence, absence of psychiatric disorders, and a 
bodyweight $\geq 35 \mathrm{~kg}{ }^{7}$ As new relevant evidence emerges, the WHO guidance will be updated and completed. ${ }^{3,10,37}$

These WHO guidelines for g-HAT treatment have a number of strengths. While previous HAT treatment guidelines relied more strongly on expert opinion and on non-systematic reviews of the evidence, ${ }^{3}$ this update followed the stricter methodology now mandatory in WHO. ${ }^{11}$ Decision-making was based on externally commissioned independent systematic reviews, and recommendations were formulated using the GRADE framework. ${ }^{15,17}$ The reviewers, methodologists and panel members all appreciated the use of the more rigorous approach as constructive.

There are some limitations that remain. ${ }^{38}$ Studies for the evaluation of treatment modalities for HAT are particularly challenging. ${ }^{39,40}$ Due to the progressive decrease in cases of g-HAT, trials cannot enrol large patient groups and have limited statistical power. ${ }^{2}$ The trials have to be conducted in remote areas in sub-Saharan Africa with a long follow-up period of 24 months. For PICO 4, adherence to oral malaria treatment was used as a proxy of the expected adherence to fexinidazole. Accordingly, the certainty of evidence supporting the recommendations were rated as either very low certainty (PICO 1, PICO 3 and PICO 4), or low certainty (PICO 2).

Regarding the question on stratification (PICO 3), the panel had to judge how much the potential benefit of avoiding lumbar puncture outweighs the inferior efficacy of fexinidazole, particularly in severe second stage. On one hand, the EMA pointed out that the decision regarding the best treatment is complex and should still rely on a combination of clinical and CSF data as currently no other equivalent method exists. ${ }^{7}$ Fexinidazole data are so far limited to a modest number of patients treated (619 patients in the three main studies DNDiFEX004-006), and there are uncertainties around factors associated with relapse, hindering proposals for less-invasive stratification. On the other hand, avoiding a lumbar puncture and CSF microscopy has other positive implications for patients and the healthcare system. ${ }^{41}$ Lumbar puncture is relatively safe, even in low resource hospitals in rural Africa, but is painful, requires adequate material and know-how, and may induce headache, back pain, confusion and in rare cases cerebral herniation. ${ }^{42}$ Fear of lumbar puncture represents a barrier to HAT screening and for seeking treatment after HAT diagnosis. ${ }^{43,44}$ The step wise approach chosen exploits the advantages of fexinidazole. A primary clinical assessment followed by a lumbar puncture only in cases of suspected severe second stage, will identify patients with high CSF leucocytosis who should receive NECT to reduce the risk of treatment failure. Indeed, neurological and psychiatric symptoms increase significantly with CSF WBCs and indicate disease progression. ${ }^{31}$

Even with the introduction of fexinidazole, systematic treatment of patients testing antibody positive in screening tests such as CATT/T.b. gambiense or in rapid diagnostic tests, but in whom no trypanosomes are detected in blood or lymph is not justified. Taking into account the limited positive predictive value of such serological tests at low prevalence, the national protocols set specific conditions for treating these patients, such as plasma titration, additional serological tests, clinical and epidemiological parameters. The national protocol may also require lumbar puncture or continued follow-up of seropositives with additional parasitological examinations. Once a patient is parasitologically confirmed or is considered as a g-HAT case based on additional criteria, the present treatment guidelines should be followed.

Fexinidazole is a new drug that has been tested only in clinical trial settings. Being a 10-day oral treatment, frequently causing nausea and vomiting, and requiring concomitant food intake for full drug absorption, there is risk of non-compliance. Hence a need for systematic patient follow-up is 
high, even if this may be challenging with limited resources. In addition, relapses with fexinidazole may occur late, up to 12 to 24 months after treatment. ${ }^{7}$ Therefore, contrary to the situation with NECT and pentamidine, where, due to their high efficacy, systematic follow-up is currently not recommended, ${ }^{3}$ patients treated with fexinidazole should return for general examination at $6,12,18$ and 24 months after treatment, or at any time if symptoms reappear. In case of signs or symptoms suggesting a possibility of relapse, laboratory examinations of body fluids, including CSF, should be performed looking for trypanosomes and CSF leukocytosis.

To date resistance to NECT has not been identified, however resistance to eflornithine and nifurtimox has been selected in the laboratory. Eflornithine resistance emerges when a transporter that carries the drug into the cell is lost $^{45}$ Nifurtimox resistance is associated with diminished activity of a nitroreductase enzyme required to activate the drug. ${ }^{46}$ The same enzyme is responsible for activation of fexinidazole and its diminished activity may cause cross-resistance between nifurtimox and fexinidazole. ${ }^{47}$ There is, therefore, a theoretical risk of resistance being selected to nifurtimox rendering parasites cross-resistant to fexinidazole, or the inverse. However, to date, the fitness of nitroreductase deficient parasites to be transmitted by tsetse flies has not been assessed. Given the mitochondrial localisation of that enzyme and prominent role of the mitochondrion in the tsetse fly stages of the parasites, it is not known whether parasites with diminished nitroreductase activity could be transmitted by tsetse flies. Furthermore, with relatively few doses of therapy currently given to HAT patients and low gambiense parasitaemias, the risk of resistance, and hence cross-resistance emerging, although theoretically possible, seems low.

Taking into account the novelty of fexinidazole to treat g-HAT, some open questions and research priorities remain. The algorithm to decide which drug to use is relatively complicated due to the higher risk for relapse observed with fexinidazole if CSF WBC $\geq 100 / \mu 1$ and to the age and body weight limitations. Risk factors for relapse after fexinidazole treatment remain poorly characterized. In this context, the development and validation of clinical scores for treatment stratification is a research priority. An ongoing study on implementation, in particular on home-based treatment and adherence will yield further information on the potential of this drug in the future (ClinicalTrials.gov Identifier: NCT03025789). Taking into account that in vitro and in vivo studies have shown that fexinidazole kills Trypanosoma brucei rhodesiense ${ }^{48}$ and that the only treatment for second stage rhodesiense HAT is the highly toxic melarsoprol, a clinical trial testing efficacy of fexinidazole to treat this form of HAT has been initiated (NCT03974178). Further studies in children aged $<6$ years or $<20 \mathrm{~kg}$ are needed to seek opportunities to improve treatment in this group.

In conclusion, fexinidazole has the potential to simplify diagnosis and treatment of g-HAT and changes clinical practice in that direction. The next steps include the incorporation of the WHO guidelines into national treatment guidelines, appropriate training of health personnel, and field implementation, as well as putting in place a pharmacovigilance system. As fexinidazole will be deployed in areas poorly served by standard pharmacovigilance systems, a pro-active data collection is required, adapted to the local field constraints. Considering the currently limited evidence, and the ongoing additional studies on fexinidazole and on acoziborole, a new, single dose oral compound for treatment of all stages of g-HAT (NCT03087955), these WHO guidelines will be updated once new results become available. 


\section{Contributors}

AKL and VL led the writing of the manuscript and contributed equally. GP and JRFM coordinated the guidelines development and led the writing of the full guidelines. EA functioned as guidelines methodologist and co-chair. GV and HB presented the systematic reviews, evidence profiles and GRADE tables. All authors contributed to the development of the New WHO Interim Guidelines for the Treatment of Human African Trypanosomiasis.

\section{Declaration of interests}

EAA, MPB, FC, AKE, OE, JRFM, LK, AKL, EM, GP, JS, PS have no COI to disclose. HB and GV report personal fees from WHO Department of Control of Neglected Tropical Diseases during the conduct of the study. VL reports personal fees from DNDi, outside the submitted work.

\section{Acknowledgments}

This paper is an abbreviated version with complementary information of the WHO Interim Guidelines for the Treatment of Human African Trypanosomiasis, which were published in August 2019. The following external peer experts reviewed the prefinal guidelines document and provided valuable input: Serena Kasparian (Médecins Sans Frontières, Montréal), Philippe Büscher (Institute of Tropical Medicine, Antwerp), Enock Matovu (University of Makerere, Uganda), and Vincent Jamonneau (Institut de Recherche pour le Développement, Montpellier). Johannes Blum (Swiss Tropical and Public Health Institute, Basel) and Peter Kennedy (University of Glasgow) provided specialist input on clinical assessment aspects. Katrin Probyn, Nicholas Henschke, Chantelle Garritty (Cochrane Response), Paul Garner, and Vittoria Lutje (Cochrane Infectious Diseases Group, UK) contributed to the systematic evidence reviews, evidence profiles and GRADE tables. Drugs for Neglected Diseases Initiative (DNDi), European Medicines Agency (EMA) and Sanofi kindly provided unpublished data for the evidence review. The development of the guidelines was supervised by a WHO steering committee and we thank the following members: Abdoulaye Diarra, Daniel Dagne, Sophie Lambert, Nicola Magrini, Lise Grout. For the discussion and conceptual validation we thank the directors and focal points of national HAT control programmes of the following disease endemic countries: Benin, Burkina-Faso, Cameroon, Congo, Ivory Coast, Gabon, Ghana, Guinea Conakry, Equatorial Guinea, Mali, Nigeria, South Sudan, Central African Republic, Democratic Republic of Congo, Chad and Togo.

Funding for the development of these guidelines was provided by WHO. 


\section{Literature}

1. WHO. Accelerating work to overcome the global impact of neglected tropical diseases - A roadmap for implementation. WHO/HTM/NTD/2012.1. Geneva: World Health Organization, 2012.

2. Franco JR, Cecchi G, Priotto G, et al. Monitoring the elimination of human African trypanosomiasis: Update to 2016. PLoS Neg/ Trop Dis 2018; 12(12): e0006890.

3. WHO. Control and surveillance of human African trypanosomiasis. Technical Report Series, no. 984. Geneva: World Health Organization, 2013.

4. Priotto G, Kasparian S, Mutombo W, et al. Nifurtimox-eflornithine combination therapy for second-stage African Trypanosoma brucei gambiense trypanosomiasis: a multicentre, randomised, phase III, non-inferiority trial. Lancet 2009; 374(9683): 56-64.

5. Eperon G, Balasegaram M, Potet J, Mowbray C, Valverde O, Chappuis F. Treatment options for second-stage gambiense human African trypanosomiasis. Expert Rev Anti Infect Ther 2014; 12(11): 1407-17.

6. Mesu V, Kalonji WM, Bardonneau C, et al. Oral fexinidazole for late-stage African Trypanosoma brucei gambiense trypanosomiasis: a pivotal multicentre, randomised, non-inferiority trial. Lancet 2018; 391(10116): 144-54.

7. European Medicines Agency. Committee for Medicinal Products for Human Use (CHMP). Assessment report. Fexinidazole Winthrop. Nov 15, 2018.

https://www.ema.europa.eu/en/documents/medicine-outside-eu/fexinidazole-winthropassessment-report en-0.pdf (accessed August 8, 2019).

8. Pelfrene E, Harvey Allchurch M, Ntamabyaliro N, et al. The European Medicines Agency's scientific opinion on oral fexinidazole for human African trypanosomiasis. PLoS Negl Trop Dis 2019; 13(6): e0007381.

9. Tarral A, Blesson S, Mordt OV, et al. Determination of an optimal dosing regimen for fexinidazole, a novel oral drug for the treatment of human African trypanosomiasis: first-in-human studies. Clin Pharmacokinet 2014; 53(6): 565-80.

10. WHO interim guidelines for the treatment of gambiense human African trypanosomiasis. Geneva: World Health Organization; 2019. https://www.who.int/neglected diseases/news/WHOpublishes-guidelines-treatment-sleeping-sickness/en/ (accessed August 8, 2019).

11. WHO. Handbook for Guideline Development. 2nd edition. Geneva: World Health Organization, 2014.

12. Huang X, Lin J, Demner-Fushman D. Evaluation of PICO as a knowledge representation for clinical questions. AMIA Annu Symp Proc 2006: 359-63.

13. Higgins JPT, Green S (editors). Cochrane handbook for systematic reviews of interventions. Version 5.1.0 [updated March 2011]. The Cochrane Collaboration, 2011. http://handbook-51.cochrane.org/ (accessed August 8, 2019).

14. Evidence summary: systematic review of oral fexinidazole as first line treatment for gambiense human African trypanosomiasis. Appendix of the WHO interim guidelines for the treatment of gambiense human African trypanosomiasis. Geneva: World Health Organization, 2019. https://www.who.int/trypanosomiasis african/resources/ISBN978-92-4-155056-7-Appendix1.pdf?ua=1 (accessed August 8, 2019).

15. Guyatt G, Oxman AD, AkI EA, et al. GRADE guidelines: 1. Introduction-GRADE evidence profiles and summary of findings tables. J Clin Epidemiol 2011; 64(4): 383-94.

16. Guyatt GH, Oxman AD, Vist $G$, et al. GRADE guidelines: 4. Rating the quality of evidencestudy limitations (risk of bias). J Clin Epidemiol 2011; 64(4): 407-15.

17. Balshem $\mathrm{H}$, Helfand $\mathrm{M}$, Schunemann $\mathrm{HJ}$, et al. GRADE guidelines: 3 . Rating the quality of evidence. J Clin Epidemiol 2011; 64(4): 401-6.

18. Andrews J, Guyatt G, Oxman AD, et al. GRADE guidelines: 14. Going from evidence to recommendations: the significance and presentation of recommendations. J Clin Epidemiol 2013; 66(7): 719-25. 
19. Burri C, Yeramian PD, Allen JL, et al. Efficacy, safety, and dose of pafuramidine, a new oral drug for treatment of first stage sleeping sickness, in a phase $2 a$ clinical study and phase $2 b$ randomized clinical studies. PLoS Negl Trop Dis 2016; 10(2): e0004362.

20. Pohlig G, Bernhard SC, Blum J, et al. Efficacy and safety of pafuramidine versus pentamidine maleate for treatment of first stage sleeping sickness in a randomized, comparator-controlled, international phase 3 clinical trial. PLoS Negl Trop Dis 2016; 10(2): e0004363.

21. Balasegaram M, Harris S, Checchi F, Hamel C, Karunakara U. Treatment outcomes and risk factors for relapse in patients with early-stage human African trypanosomiasis (HAT) in the Republic of the Congo. Bull World Health Organ 2006; 84(10): 777-82.

22. Bastide S, Priotto G, Echochard R, Etard J. Effectiveness of short vs. long treatment schedules with pentamidine in first-stage HAT: a large field cohort study. Tropical Medicine and International Health 2011; 16: 68-9.

23. Doua F, Yapo FB. Human trypanosomiasis in the Ivory Coast: therapy and problems. Acta Trop 1993; 54(3-4): 163-8.

24. Eperon G, Schmid C, Loutan L, Chappuis F. Clinical presentation and treatment outcome of sleeping sickness in Sudanese pre-school children. Acta Trop 2007; 101(1): 31-9.

25. Ginoux PY, Bissadidi N, Frezil JL. [Complications observed in the treatment of trypanosomiasis in the Congo]. Med Trop (Mars) 1984; 44(4): 351-5.

26. Jamonneau V, Solano P, Garcia A, et al. Stage determination and therapeutic decision in human African trypanosomiasis: value of polymerase chain reaction and immunoglobulin $\mathrm{M}$ quantification on the cerebrospinal fluid of sleeping sickness patients in Cote d'Ivoire. Trop Med Int Health 2003; 8(7): 589-94.

27. Mumba Ngoyi D, Lejon V, Pyana P, et al. How to shorten patient follow-up after treatment for Trypanosoma brucei gambiense sleeping sickness. J Infect Dis 2010; 201(3): 453-63.

28. Ruiz JA, Simarro PP, Josenando T. Control of human African trypanosomiasis in the Quicama focus, Angola. Bull World Health Organ 2002; 80(9): 738-45.

29. Tongue Kohagne L, Louis F, Dologuele N. Relapse after treatment with first stage drug in human African trypanosomiasis: contribution of molecular biology. International Journal of Infectious Diseases 2008; 12(1).

30. Anne Loes van Staa AH. Injection practices in the developing world - results and recommendations from field studies in Uganda and Indonesia - EDM Research Series No. 020, 1996. 31. Blum J, Schmid C, Burri C. Clinical aspects of 2541 patients with second stage human African trypanosomiasis. Acta Trop 2006; 97(1): 55-64.

32. Kennedy PG. Clinical features, diagnosis, and treatment of human African trypanosomiasis (sleeping sickness). Lancet Neurol 2013; 12(2): 186-94.

33. Banek K, Lalani M, Staedke SG, Chandramohan D. Adherence to artemisinin-based combination therapy for the treatment of malaria: a systematic review of the evidence. Malar $J$ 2014; $13: 7$.

34. Bruxvoort K, Goodman C, Kachur SP, Schellenberg D. How patients take malaria treatment: a systematic review of the literature on adherence to antimalarial drugs. PLoS One 2014; 9(1): e84555.

35. Fuangchan A, Dhippayom T, Kongkaew C. Intervention to promote patients' adherence to antimalarial medication: a systematic review. Am J Trop Med Hyg 2014; 90(1): 11-9.

36. Yakasai AM, Hamza M, Dalhat MM, et al. Adherence to artemisinin-based combination therapy for the treatment of uncomplicated malaria: a systematic review and meta-analysis. $J$ Trop Med 2015; 2015: 189232.

37. WHO. The selection and use of essential medicines: report of the WHO Expert Committee, 2015 (including the 19th WHO model list of essential medicines and the 5th WHO model list of essential medicines for children). WHO technical report series, no. 994. Geneva: World Health Organization, 2015.

38. Norris SL, Ford N. Improving the quality of WHO guidelines over the last decade: progress and challenges. Lancet Glob Health 2017; 5(9): e855-e6. 
39. WHO. Human African trypanosomiasis: update of the methodological framework for clinical trials. Report of the first meeting of the development of new tools subgroup.

WHO/HTM/NTD/IDM/2015.5. Geneva: World Health Organization, 2014.

40. WHO. Recommendations of the informal consultation on issues for clinical product development for human African trypanosomiasis. WHO/CDS/NTD/IDM/2007.1. Geneva: World Health Organization, 2007.

41. Chappuis F. Oral fexinidazole for human African trypanosomiasis. Lancet 2018; 391(10116): $100-2$.

42. Mukendi D, Kalo JL, Kayembe T, et al. Where there is no brain imaging: Safety and diagnostic value of lumbar puncture in patients with neurological disorders in a rural hospital of Central Africa. J Neurol Sci 2018; 393: 72-9.

43. Mpanya A, Hendrickx D, Vuna M, et al. Should I get screened for sleeping sickness? A qualitative study in Kasai province, Democratic Republic of Congo. PLoS Negl Trop Dis 2012; 6(1): e1467.

44. Mpanya A, Hendrickx D, Baloji S, et al. From health advice to taboo: community perspectives on the treatment of sleeping sickness in the Democratic Republic of Congo, a qualitative study. PLoS Negl Trop Dis 2015; 9(4): e0003686.

45. Vincent IM, Creek D, Watson DG, et al. A molecular mechanism for eflornithine resistance in African trypanosomes. PLoS Pathog 2010; 6(11): e1001204.

46. Wilkinson SR, Taylor MC, Horn D, Kelly JM, Cheeseman I. A mechanism for cross-resistance to nifurtimox and benznidazole in trypanosomes. Proc Natl Acad Sci U S A 2008; 105(13): 5022-7.

47. Wyllie S, Foth BJ, Kelner A, Sokolova AY, Berriman M, Fairlamb AH. Nitroheterocyclic drug resistance mechanisms in Trypanosoma brucei. J Antimicrob Chemother 2016; 71(3): 625-34.

48. Kaiser M, Bray MA, Cal M, Bourdin Trunz B, Torreele E, Brun R. Antitrypanosomal activity of fexinidazole, a new oral nitroimidazole drug candidate for treatment of sleeping sickness. Antimicrob Agents Chemother 2011; 55(12): 5602-8. 


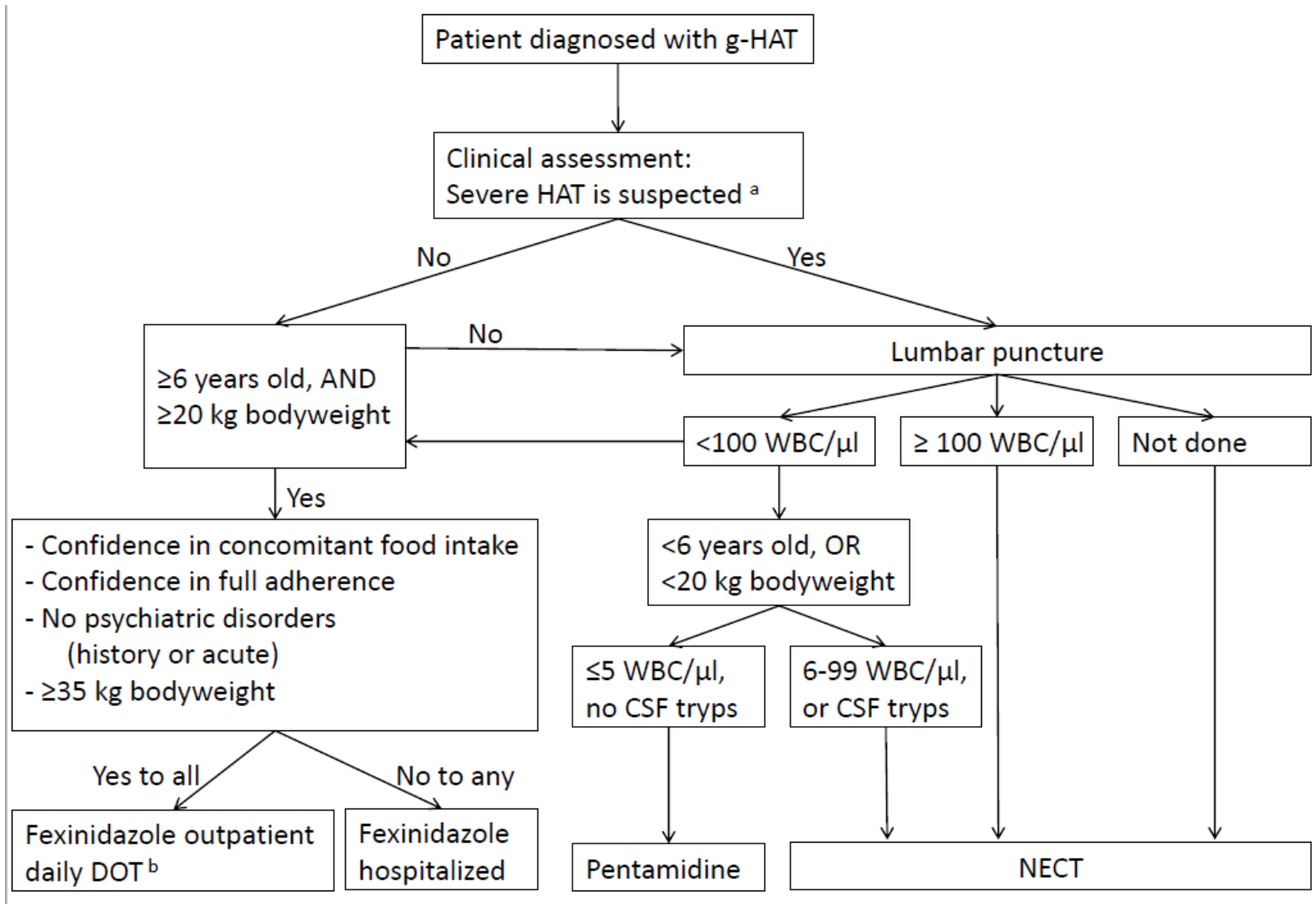

Figure 1: Algorithm of WHO guidelines for the management of persons with gambiense HAT.

a Presence of any symptom or sign consistent with severe second-stage g-HAT, detailed in table 2;

${ }^{b}$ If the health facility has capacity for supervised administration as outpatient. DOT: directly observed therapy 


\begin{tabular}{|c|c|c|c|c|}
\hline Population & Intervention/comparator & Recommendation favouring & $\begin{array}{l}\text { Strength of } \\
\text { recommendation } \\
\text { (certainty of evidence) }\end{array}$ & Key considerations \\
\hline Patients with first stage g-HAT & Fexinidazole or pentamidine & $\begin{array}{l}\text { Use of fexinidazole over } \\
\text { pentamidine }\end{array}$ & Conditional (very low) & $\begin{array}{l}\text { Children }<6 \text { years old or }<20 \mathrm{~kg} \text { should receive } \\
\text { pentamidine, as fexinidazole is not approved for } \\
\text { this group. }\end{array}$ \\
\hline $\begin{array}{l}\text { Patients with second stage g- } \\
\text { HAT if } C S F<100 \mathrm{WBC} / \mu \mathrm{l}\end{array}$ & Fexinidazole or NECT & Use of fexinidazole over NECT & Conditional (low) & $\begin{array}{l}\text { Children }<6 \text { years old or }<20 \mathrm{~kg} \text { should receive } \\
\text { NECT, as fexinidazole is not approved for this } \\
\text { group. }\end{array}$ \\
\hline $\begin{array}{l}\text { Patients with second stage g- } \\
\text { HAT if CSF } \geq 100 \mathrm{WBC} / \mu \mathrm{ll} \\
\text { (severe second stage) }\end{array}$ & Fexinidazole or NECT & Use of NECT over fexinidazole & Conditional (low) & $\begin{array}{l}\text { Especially in severe second stage the risk of } \\
\text { treatment failure is higher with fexinidazole. }\end{array}$ \\
\hline $\begin{array}{l}\text { Patients diagnosed with } \mathrm{g} \text { - } \\
\text { HAT, prior to treatment }\end{array}$ & $\begin{array}{l}\text { Stratification by clinical exam or by } \\
\text { CSF exam or no stratification }\end{array}$ & $\begin{array}{l}\text { A lumbar puncture/CSF exam in } \\
\text { case of clinical signs and symptoms } \\
\text { raising suspicion of severe second } \\
\text { stage g-HAT } \\
\text { AND } \\
\text { No lumbar puncture in the absence } \\
\text { of such clinical suspicion }\end{array}$ & Conditional (very low) & $\begin{array}{l}\text { In the absence of clinical suspicion of severe } \\
\text { second stage g-HAT, a lumbar puncture can be } \\
\text { avoided and fexinidazole preferentially be given in } \\
\text { case of high confidence in appropriate follow-up to } \\
\text { detect relapse early. There are no validated clinical } \\
\text { scores for patient stratification and their } \\
\text { development is a research priority. Children }<6 \\
\text { years old or }<20 \mathrm{~kg} \text { require a lumbar puncture (as } \\
\text { fexinidazole is not approved for this group). }\end{array}$ \\
\hline $\begin{array}{l}\text { Patients requiring } \\
\text { fexinidazole administration }\end{array}$ & $\begin{array}{l}\text { Inpatient or outpatient } \\
\text { administration of fexinidazole }\end{array}$ & $\begin{array}{l}\text { Fexinidazole to be given in } \\
\text { outpatient setting when all of the } \\
\text { following conditions are met: } \\
\text { confidence in concomitant food } \\
\text { intake; confidence in full } \\
\text { adherence; absence of psychiatric } \\
\text { disorders (history or acute); } \\
\text { bodyweight } 235 \mathrm{~kg}\end{array}$ & $\begin{array}{l}\text { Conditional for either } \\
\text { inpatient or outpatient } \\
\text { (verylow) }\end{array}$ & $\begin{array}{l}\text { Outpatient administration should be done in } \\
\text { hospitals or peripheral health facilities, and in } \\
\text { particular situations, at home, but always under } \\
\text { the strict supervision of trained health staff to } \\
\text { ensure daily compliance of drug intake with food } \\
\text { (given the insufficient drug absorption without } \\
\text { food) }\end{array}$ \\
\hline
\end{tabular}




\begin{tabular}{|c|c|}
\hline Mental confusion: & $\begin{array}{l}\text { disorientation in time and/or space, inattention, slowed thought processes, memory } \\
\text { problems }\end{array}$ \\
\hline $\begin{array}{l}\text { Abnormal } \\
\text { behaviour: }\end{array}$ & $\begin{array}{l}\text { manifestly abnormal, inappropriate and/or unusual behaviour for that patient, such as } \\
\text { disinhibition, excitement, euphoria, aggressiveness, or indifference }\end{array}$ \\
\hline Logorrhoea: & excessive, incontrollable or incoherent speech \\
\hline Speech impairment: & inability to speak and articulate words normally \\
\hline Anxiety: & worried about every life event \\
\hline Tremor: & involuntary twitching movements of one or more body parts \\
\hline Motor weakness: & weakness in one or more muscles groups, usually the limbs and/or trunk \\
\hline Ataxia: & $\begin{array}{l}\text { loss of muscle control in the arms and legs, which may lead to a lack of balance, } \\
\text { coordination, and possibly a disturbance of gait. Ataxia may affect the fingers, hands, } \\
\text { arms, legs, body, speech, and even eye movements }\end{array}$ \\
\hline Abnormal gait: & patient cannot walk normally \\
\hline $\begin{array}{l}\text { Abnormal } \\
\text { movements: }\end{array}$ & $\begin{array}{l}\text { uncontrollable and abnormal movements which can affect the limbs, trunk, face or neck, } \\
\text { such as facial grimacing, tremor, chorea and/or athetosis in the limbs, that also may be } \\
\text { observed during walking or on clinical examination }\end{array}$ \\
\hline Seizures: & $\begin{array}{l}\text { uncontrolled shaking movements involving much of the body with loss of consciousness } \\
\text { (tonic-clonic seizure), or shaking movements involving only part of the body with variable } \\
\text { levels of consciousness (focal seizure) }\end{array}$ \\
\hline
\end{tabular}

Table 2: The presence of any of these symptoms or signs should raise suspicion of severe second stage g-HAT (i.e. CSF WBC $\geq 100 / \mu \mathrm{l}$ ). 